\title{
Interfaces
}

\section{Travailler avec/contre le format - Éthique et esthétique dans l'œuvre avec figures de Stanley Spencer}

\section{Liliane Louvel}

\section{OpenEdition}

Journals

\section{Édition électronique}

URL : https://journals.openedition.org/interfaces/2435

DOI : 10.4000/interfaces.2435

ISSN : 2647-6754

Éditeur :

Université de Bourgogne, Université de Paris, College of the Holy Cross

Édition imprimée

Date de publication : 12 juillet 2021

ISSN : 1164-6225

Référence électronique

Liliane Louvel, «Travailler avec/contre le format - Éthique et esthétique dans l'œuvre avec figures de Stanley Spencer », Interfaces [En ligne], 45 | 2021, mis en ligne le 12 juillet 2021, consulté le 15 septembre 2021. URL : http://journals.openedition.org/interfaces/2435 ; DOI : https://doi.org/ $10.4000 /$ interfaces. 2435

Ce document a été généré automatiquement le 15 septembre 2021.

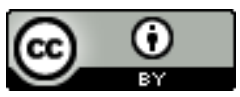

Les contenus de la revue Interfaces sont mis à disposition selon les termes de la Licence Creative Commons Attribution 4.0 International. 


\title{
Travailler avec/contre le format - Éthique et esthétique dans l'œuvre avec figures de Stanley Spencer
}

\author{
Liliane Louvel
}

\section{Le choix de la forme où inscrire l'imaginaire du peintre $^{1}$}

1 La question inaugurale du format adéquat de la toile sur laquelle le peintre va tracer les premiers traits de la composition, installer ou non la « couverture » et la sous-couche puis travailler la couleur a une valeur programmatique héritée de l'ère classique et académique. Chez le "marchand de couleurs », les formats étaient répertoriés selon les sujets : peinture d'histoire, portrait, figure, marine, paysage, etc. Le fait de se plier ou non à cette forme de calibrage était déjà en soi un choix esthétique et éthique. Victor Stoichita rappelle comment Manet, choisissant le grand format, celui du grand genre, jusque-là réservé aux toiles dans la tradition des sujets classés en haut de la hiérarchie de la peinture (sujets religieux, sujets historiques ou mythologiques avec figures), « se confrontait à la fois à la tradition et à l'art contemporain [...]. Manet préfère [...] les toiles de grand format propres au Salon » (Stoichita 2015, 28). Choisir le format noble pour y peindre un sujet trivial devenait alors une provocation et une transgression, au moment où les Impressionnistes, quant à eux, préféraient un format plus intime. Le choix d'un format prenait alors la forme d'un enjeu, celui d'un manifeste esthétique. Ne pas le respecter impliquait que l'artiste lançait un défi à l'ordre établi par les autorités de régie et, en même temps, affirmait la liberté de sa position individuelle, hors normes, comme l'était le choix esthétique de son mode de (re)présentation. De ce fait, il se plaçait clairement en rupture avec la tradition.

2 On peut dire qu'une pragmatique du format a été clairement posée par David Zerbib: «Que font les formats? Ils définissent des conditions d'expérience, des cadres de perception, des possibilités pratiques, des modes de connaissance, de représentation ou 
de construction du réel » (Zerbib 2015b, 21). Il ajoute que les questions d'échelle, de dimensions, de diagramme, font aussi subir à des textes, des images, certaines opérations pragmatiques de traduction, de remédiation, par exemple ${ }^{2}$. Toutes pratiques intermédiales (Louvel 2018).

3 Stanley Spencer (1891-1959) est un peintre britannique de la première moitié du XX siècle parmi les plus connus dans son pays mais peu en France, et dont on peut dire qu'il est hors normes. Hors des normes de la peinture "classique », d'une part, mais aussi hors des normes de la révolution artistique qui s'est effectuée au tournant de la modernité picturale du $\mathrm{XX}^{\mathrm{e}}$ siècle amorcée par les Impressionnistes et les peintres du continent européen. Viendraient ensuite le post-impressionnisme, l'expressionnisme, le cubisme, le surréalisme, le fauvisme... tous mouvements et écoles de peinture bien connus. Ses toiles avec figures ont même été qualifiées d'anormales, « abnormal », par la critique souvent choquée voire "repoussée » par elles, le terme de "repellent» ayant même été utilisé (Louvel 2017b). En août 1936, dans un article anonyme de The Apollo, on pouvait lire: "Stanley Spencer is a genius if 'an abnormal one' " (Bell 1992, 162). Chez Spencer, la matière picturale, le choix des sujets et l'utilisation des formats permettent d'observer comment il occupe une place tout à fait originale dans son époque, comme « décalée » temporellement.

Stanley Spencer naquit en 1891 à Cookham, petit village du Berkshire dans le sud-ouest de Londres, sur l'une des berges de la Tamise. Issu d'une famille de huit enfants tous élevés par leurs parents puis par leur sœur aînée dans le cadre familial, il garda un attachement profond au lieu ${ }^{3}$. Le père, 'Pa, était organiste et certains de ses fils, dont Will, devinrent d'excellents musiciens. Spencer était lui aussi sensible à la musique mais il se tourna vers la peinture comme son frère puiné, Gilbert. Cependant, dans certaines de ses lettres, il déclare s'inspirer de la forme de la fugue pour la composition : suites et variations... Il parvint, grâce à une bourse, à aller étudier à la Slade School of Fine Art de Londres, de 1909 à 1912, à un moment charnière dans l'histoire de la peinture en plein renouveau. Mais, sous l'influence de son maître de dessin, Henry Tonks, Spencer choisira de ne pas participer pleinement à la révolution post-impressionniste et cubiste même si l'on peut en déceler des traces dans certaines de ses toiles originelles. S'inspirant davantage des maitres anciens, il appréciait surtout les Primitifs italiens. Spencer resta à l'écart de ses confrères même s'il en côtoya certains comme Henry Lamb, la famille Carline, et le "groupe de Hampstead», et même, épisodiquement, le groupe de Bloomsbury. Il choisit de vivre à Cookham et d'allier son éthique du religieux à ses choix esthétiques ${ }^{4}$. La question du choix du format et les hasards des commandes privées allaient lui permettre de donner le cadre et la mesure nécessaires pour y inscrire et y faire tenir son œuvre. Stanley Spencer fait partie de cette génération qui subit deux guerres mondiales. Lui et ses frères s'engagèrent dans la première et en revinrent diversement meurtris. Gilbert et Stanley furent victimes du paludisme et Sydney, le frère aîné ne revint pas. La guerre et ses suites allaient donner à Spencer l'occasion de peindre l'œuvre qui allait le rendre célèbre : les fresques de la chapelle de Burghclere. La Seconde Guerre mondiale allait aussi lui donner, en tant que peintre officiel, l'occasion de se rendre sur les chantiers navals de la Clyde et d'y peindre les scènes de l'effort de guerre. Ces œuvres-là sont conservées à Londres, à l'Imperial War Museum ${ }^{5}$. Dans ces dernières, ainsi que dans ses œuvres domestiques, la question du choix du format pour rendre sa vision fut cruciale pour Spencer. 
5 Le format, a fortiori s'il est récurrent chez un peintre, participe de sa vision, de son imaginaire et de son éthique personnelle. Chez Spencer ce sera bien le cas. On peut y voir, comme chez Manet, une forme de manifeste artistique et éthique. Spencer affectionnait des formats oblongs lui permettant de travailler sur la longueur, d'articuler ses figures, de répéter et de varier les thèmes en des scènes et des séries frappantes. Le format unifie, évite le discontinu, contient les figures, même si parfois il en déborde. "La suite et la série supposent le mouvement inverse d'un ensemble de choses hétérogènes et séparées qui tendent, dans le mouvement de la suite ou de la série, vers une certaine unité » (Vaugeois 1998, 311). Pour Spencer, le double choix du format, allié à celui de la série, lui permettait d'instaurer et de donner à voir un lieu imaginaire manifestant ainsi l'alliance entre le transcendantal nourri du religieux et l'immanent de la vie terrestre. La fusion entre les deux plans s'incarne au mieux dans le choix constant d'adopter comme décor récurrent de ses scènes d'inspiration religieuse son village natal de Cookham, dans le Berkshire, dans lequel il voyait à la fois un village anglais traditionnel et rassurant (ce qu'il est encore de nos jours) et un "paradis sur terre ». Ses camarades de la Slade School of Fine Art de Londres, le surnommèrent «Cookham » et c'est ainsi qu'il adopta le sobriquet, signant ses lettres de ce nom de lieu.

6 Le format d'un tableau est plus qu'un cadre avec ses dimensions variées et codifiées, il est un outil esthétique majeur permettant au peintre de projeter son monde intérieur imaginaire. Pour Spencer, il s'agissait de trouver le lieu propre à inscrire des scènes d'une agitation foisonnante, empreinte d'une inspiration toute médiévale qui lui fournissait ses modèles favoris et des souvenirs de tableaux contemplés et assimilés dont il donne de nouvelles versions ${ }^{6}$. Le choix d'un format donne alors place à son imaginaire; celui d'un certain anachronisme aussi. L'une des marques de fabrique de Spencer, dans ses tableaux à figures, fut la distorsion qu'on lui a souvent reprochée. Cependant, il s'agissait là encore d'un choix principe esthétique nécessaire clairement revendiqué : "I do myself love the 'disagreeably abnormal persons in these paintings' » (Bell 1980, 168) affirme-t-il dans une lettre à Hilda, sa première femme, en 1944. Il s'agissait bien d'une vision à transmettre, ce qu'il nommait " his inner vision» (168), et non pas d'un servile procédé mécanique dont il pensait qu'il était cependant à l'œuvre dans ses tableaux de paysages, de natures mortes et de portraits résultant souvent de commandes (168). Dans ces productions alimentaires, il disait ne voir que de simples reproductions de ce qu'il avait eu sous les yeux en réalité. Même si les choses sont loin d'être aussi simples, bien entendu, puisque ces œuvres sont aussi particulièrement réussies et inspirèrent plus tard de jeunes artistes. Cependant, je m'intéresserai ici surtout aux tableaux à figures de ce peintre étonnant, car ce sont elles qui le distinguent et lui donnèrent l'occasion d'expérimenter la question du format d'une manière stupéfiante. C'est là aussi où nous retrouverons comme une nécessité, le choix de l'agencement en séries ainsi que celui des formes canoniques du triptyque et du polyptyque, tous correspondant à des "dispositifs » étonnants comme définis par Philippe Ortel (Ortel 2008). Dominique Vaugeois, quant à elle, voit dans la série une manière de saisir le «style » d'un artiste :

C'est d'ailleurs à travers à travers cette notion de série que se rejoignent pour Merleau-Ponty le peintre et l'écrivain, autour de la notion centrale d'expression et de style, la loi de saisie de l'expression passant pour le peintre comme pour l'écrivain par un pas à pas de tableau en tableau, de texte en texte, et pour le spectateur-et cela vaut pour le lecteur-c'est dans la série des tableaux que peut se 
saisir « le style » du peintre qui est, on le sait, pour le philosophe, l'essentiel de l'art, c'est-à-dire l'expression du rapport particulier d'un homme au monde. (Vaugeois $310)^{7}$

7 Ainsi, la série, entendue comme dispositif génératif d'une œuvre et aussi comme moyen de percevoir le style d'un artiste par l'amateur, se découvre comme procédé esthétique à double détente. À l'origine de l'œuvre, du côté du créateur, le choix du dispositif permet d'inscrire et de produire l'œuvre. Parallèlement, du côté du lecteur et de la réception, il est un moyen de reconnaître et d'identifier l'expression d'une singularité et d'un style. C'est ainsi que chez Spencer, le lieu inspirera le format de l'œuvre lorsque se présentera l'opportunité de décorer les murs d'une chapelle. Il pourra alors mettre en œuvre une pratique anachronique féconde.

\section{L'influence des Primitifs italiens : un peintre de fresques au $\mathrm{XX}$ siècle}

En regardant l'œuvre à figures de Spencer, on constate qu'il privilégiait l'utilisation de longs formats étroits (Causey 29), parfois des œuvres faisant plus de cinq, voire, six mètres de long. C'est qu'il aimait s'immerger dans son œuvre, s'engager pour un laps de temps assez long, et comme vivre dans un espace imaginaire, entouré de ses "figures » auxquelles il prêtait une identité et une forme de vie. L'un de ses amis peintres de la Slade, Paul Nash, se souvint que les premières œuvres de Spencer étaient pour lui : « the real thing with all the right inheritance of the fine qualities of the mighty [...] which caught one by a strange enchantment" (Abbott et Bertram 154 cités par Causey 22). Saisi "d'un étrange enchantement » : comment mieux exprimer la capture du spectateur face à une fascinante vision, celle d'un ailleurs insolite soudain rendu visible?

Toute sa vie, Spencer cultiva une profonde admiration pour les peintres primitifs italiens (Martin 53-63), Giotto d'abord mais aussi Piero della Francesca, Fra Angelico, Masaccio, Uccello... À la Slade School of Fine Art, le groupe auquel il appartint un temps s'était appelé «The Primitives ». Dans les éditions du Who's Who in Art de 1929 et 1934, Stanley se décrit simplement comme "peintre de fresques", "a mural painter", insistant ainsi sur l'osmose nécessaire, pour lui, entre l'œuvre et la technique (Causey 29). Il reprenait ainsi l'héritage de la peinture ancienne religieuse, celle d'avant «l'instauration du tableau " (Stoichita 1999). Peignant une scène, un événement, il pensait que les protagonistes se réalisaient mieux lorsqu'ils étaient en accord avec des lieux qui leur convenaient. Il y avait là pour lui comme une nécessité intime et profonde. Et ce fut dans ces années-là qu'il eut la chance, juste après la Première Guerre mondiale, de pouvoir réaliser son rêve : peindre une chapelle sur le grand modèle de la chapelle de l'Arena de Padoue peinte à fresque par Giotto.

Dans les années vingt, la famille Berhend, qui possédait déjà Swan Upping in Cookham $(1919)^{8}$, lui passa commande de "fresques » pour décorer l'intérieur de leur futur Mémorial dédié au Lieutenant Sandham, le frère de Mrs Berhend, mort des suites de la guerre 9 . C'est ainsi qu'il put réaliser l'intérieur du fameux Sandham Memorial de Burghclere, dans le Hampshire. Cette chapelle, baptisée Chapel of All Souls, est décorée sur le modèle des chapelles à fresques distribuées sur trois niveaux, culminant dans la grande "fresque » du fond, la pala d'autel, dédiée à The Resurrection of the Soldiers. Elle montre à quel point la forme, les dimensions, le lieu, ont conditionné la composition. Spencer l'a conçue en fonction de l'architecture de la chapelle et a donc divisé les deux 
murs latéraux en trois bandes inégales, elles-mêmes divisées en scènes, sur le modèle des retables: au niveau inférieur des prédelles, la vie de tous les jours à l'hôpital Beaufort de Bristol, où il avait travaillé comme infirmier; au niveau intermédiaire, de grands formats aux sommets courbes dédiés essentiellement à sa vie comme brancardier puis soldat sur le «front d'Orient $»^{10}$ en Macédoine et en Bulgarie; audessus, surplombant chaque côté, se déroule une longue fresque de forme oblongue plus petite et dédiée à la vie des soldats dans les camps de Todorov et de Karasuli. Les œuvres des côtés sont réalisées sur des panneaux peints à l'huile. Seules les scènes supérieures et la grande Resurrection of the Soldiers ont été peintes sur le mur ${ }^{11}$.

11 Dans les retables religieux les registres se déploient ainsi : au niveau inférieur, la relation de la vie ordinaire des saints et des humains ; au niveau médian, des scènes sacrées mettant en scène la Vierge, le Christ et les saints ; au-dessus apparaissent Dieu ou Son fils, et souvent le Saint Esprit sous forme de colombe. Bien sûr il y a des variantes (Chastel 70, 112) ${ }^{12}$. Cependant, à Burghclere, dans les trois registres, ce sont des scènes de la vie des blessés, des soignants et des soldats, qui sont représentées. L'alliance du format, d'ordinaire réservé aux sujets religieux et ici dédié à des thèmes empruntés au quotidien, aussi poignant et concret soit-il, finalement, conférait à ces hommes le statut de héros ou de quasi-saints. Cependant, un thème religieux récurrent tout au long de la vie du peintre, celui de La Résurrection, est lui, pleinement assumé dans la pala d'autel, la grande Résurrection des soldats aux dimensions impressionnantes : $6,40 \times 5,26 \mathrm{~m}$. Le format de l'œuvre a été adapté à celui du mur percé de deux portes sur lequel elle est peinte. Elle se déploie derrière l'autel et monte jusqu'en haut du mur. Là encore, il y a trois niveaux. Les têtes des soldats prises dans l'entrecroisement des croix apparaissent juste au-dessus de l'autel au milieu des croix blanches qui ont été érigées sur leurs tombes. Au second niveau, la révélation de la résurrection en train d'avoir lieu rejoint le troisième niveau de la scène et du tableau, et culmine avec l'apparition du Christ. À noter que ce dernier n'est pas de dimension supérieure à celles des soldats. Le format d'un retable est repris, varié, adapté dans cette instanciation. Le fait que Spencer choisisse de ne pas davantage distinguer le Christ des soldats va à l'encontre de la pratique médiévale qui modulait les dimensions des personnages selon leur importance sacrée. S'ils figuraient bien dans le triptyque, les donateurs et leur descendance étaient relégués dans les marges et représentés dans un format très modeste (Louvel 2017a).

\section{« The Church House Project » et ses variantes sérielles}

La réussite de cet énorme travail solitaire effectué à Burghclere, qui avait duré cinq ans de 1927 à 1932, avait si bien convenu à l'artiste qu'il souhaita continuer à travailler de cette manière, plongé comme en immersion totale à la fois dans un lieu et dans une œuvre. L'idée d'une suite à donner lui était venue lors de la construction des deux petits hospices ajoutés de chaque côté de la chapelle. Il conçut alors un projet qu'il nomma "The Church House Project», programme de fresques qui devait encore trouver un lieu adéquat et surtout, un commanditaire. Malheureusement le projet ne trouva jamais ni l'un ni l'autre et resta inachevé. Cependant, Spencer ne cessa d'y travailler en créant des œuvres qui répondaient à cette vision si personnelle et en conçut, tout au long de sa vie, des variantes proprement extraordinaires, espérant toujours mener à bien son projet. Il s'agissait pour lui de mêler à ses visions imaginaires 
une version de la vie qui, parfois aussi, frise l'autobiographie. La fusion du religieux, exprimé par le mot "Church", et de la vie de tous les jours, présente dans " House ", devait culminer dans un nouveau rêve de pierre et de peinture, à l'instar de Burghclere. On retrouve là l'un des traits de caractère du peintre, marqué par son éducation dans la maison de Cookham, «Fernlea», où le sacré et l'éducation religieuse étaient intimement mêlés à la vie quotidienne de la famille et à sa vision du monde. «In Cookham, he saw 'Jerusalem in England's green and pleasant land'» écrit Elizabeth Rothenstein (6), une amie de Spencer ${ }^{13}$. Plus loin, elle résume ainsi la personnalité complexe du peintre: "His nature, however, essentially that of a medieval crafstman and village boy, a nature of rare purity containing both intuitive and religious powers, has come into contact with the modern world» (7). Dans le désir de ce lieu à double fonction publique/ intime, se reflète enfin un autre de ses traits de caractère : l'aspiration à retrouver la protection du cocon familial où il avait été si heureux, le fameux paradis de Cookham.

13 Pour le bâtiment, il avait conçu un vrai programme iconographique, commençant par en dessiner certaines parties puis les matérialisant sous forme de maquette réalisée dans du carton découpé ${ }^{14}$. On connaît le caractère prospectif de ce type de reproduction à une échelle inférieure d'un objet architectural absent (During 24). Dans ses écrits, l'on voit qu'il avait clairement envisagé une nef, un transept et des chapelles adjacentes. Il s'agissait bien, là encore, de concevoir une œuvre totale joignant la décoration à l'architecture d'un lieu. Les œuvres devaient être conçues en fonction de ce format-là. Il projetait alors de célébrer la vie quotidienne à Cookham et de montrer ses habitants se promenant sur les murs de la nef et du transept. Une Pentecôte devait descendre du haut de la nef vers les côtés. Des chapelles latérales étaient dédiées aux femmes de sa vie, Hilda et Elsie, leur bonne, Patricia Preece et Daphne Charlton. Les nus de Patricia Preece et leurs doubles portraits à tous deux, si remarquables dans leur traitement, devaient aussi à l'origine y être accrochés ${ }^{15}$.

C'est alors que, dans ces différentes propositions, on voit se confirmer le goût de Spencer pour le format de la série, de la reprise et de la variation d'un sujet jusqu'à épuisement ou bifurcation de celui-ci (Moncond'huy et Noudelmann). C'était là, pour Spencer, un moyen de développer un thème, voire une histoire, et de satisfaire son goût pour la narration. Il en donnait ainsi une illustration loin d'être servile et répondait au reproche formulé par Roger Fry qui ne cessa de l'affecter (Fry cité dans Bell 1992, 67) ${ }^{16}$. Ce goût de la répétition thématique se constituant en série, se moulant dans les mêmes formats, sera constant jusqu'à la fin de sa vie. Dans le cas de "The Church House Project ", on en trouve de nombreuses instances: The Pentecost, The Marriage at Cana ${ }^{17}$, The Baptism of Christ, toutes regroupées sous le titre général de The Last Day ou The Last Judgement (Bell 1980, 122). La série des Noces de Cana comporte deux cycles célébrant la vie conjugale pour le premier, paradoxalement au moment où Spencer allait divorcer de Hilda, et la sexualité pour le second. La première série, intitulée Domestic Scenes (1935-1936), comporte neuf tableaux montrant un couple ayant assisté au baptême du Christ, rentrant se changer ou ayant assisté au mariage, et se préparant à aller se coucher ${ }^{18}$. Y entrent des œuvres comme At The Chest of Drawers, Choosing a Petticoat, On the Landing entre autres, dans lesquelles un homme de très petite stature, ce qui était le cas de Spencer, est comme écrasé par une femme aux formes amples, tubulaires et gonflées. Les œuvres de cette série sont de formats modestes : $51 \times 76 \mathrm{~cm}$ ou $66 \mathrm{~cm}$ pour la plupart. 

la passion malheureuse du peintre pour sa seconde femme Patricia Preece et au désir sexuel bouleversant mais non satisfait de retour. Il présente des couples dans des attitudes toutes de distorsions et de contorsions, avec, de nouveau, un homme petit contemplant, adorant, vénérant une femme dominatrice. La déclinaison des titres (Passion or Desire, Worship, Contemplation, Romantic Meeting, etc. ${ }^{19}$ ) dit assez à quel point la série a développé la radicalité du sujet ouvertement sexuel et semblant s'éloigner des "Béatitudes ». Ces tableaux devaient être accrochés dans de petites chapelles annexes réservées à la méditation sur l'amour et le sexe (Bell 1980, 166). Ils appartenaient aussi à la série du Mariage à Cana. Les dimensions en sont par exemple $76,2 \times 50,8 \mathrm{~cm}$, pour Passion or Desire; $91,4 \times 73,7 \mathrm{~cm}$ pour Worship; $91,4 \times 61 \mathrm{~cm}$ pour Contemplation, soit des formats identiques ou légèrement plus grands que ceux des scènes domestiques. des œuvres de la série du Baptême du Christ ont été fondues dans les Noces de Cana comme La Promenade des femmes (1938) ou A Village in Heaven (1937). Une autre série devait aussi prendre place dans la nef de la maison-église sur le thème du Jugement dernier ou plutôt de The Last Day que Spencer préférait au premier qu'il jugeait trop sévère dans la nette séparation entre les justes et les damnés sans possibilité de rédemption. Il témoignait là d'une vision particulièrement bienveillante de l'humanité. Aux trois projets réunis sous la bannière de The Last Day, il ajoute A Village in Heaven (45,7×182,9 cm, 1937), la série des Adorations qui déroule la vie du village dans une célébration de l'amour libre avec des attitudes débridées dans Adoration of Girls (92×135,9 cm, 1937), The Village Lovers $(69,9 \times 105,6 \mathrm{~cm}, 1937)$ et Adoration of Old Men $(92,7 \times 111,8 \mathrm{~cm}, 1937)$. On voit alors que les formats ont tendance à s'allonger en des bandes narratives oblongues de plus d'un mètre, ce qui annonce une mutation vers une nouvelle utilisation du format.

Dans ces œuvres, grouillantes de personnages affairés affichant des expressions et des attitudes outrées ou exacerbées ainsi que des distorsions physiologiques, on perçoit l'influence des sculptures se déployant sur les façades des temples hindous tant admirées par Spencer. Il va donc, lorsque ce sera nécessaire, multiplier les figures sur des bandes de trois, quatre, voire six mètres de long, ce qui requiert du spectateur un déplacement le long de la toile, un peu comme dans les galeries de peinture des châteaux ou encore devant les rouleaux de peinture chinoise alignant diverses séquences. Alors, c'est le spectateur qui construit le discours de l'histoire (Moncond'huy 21-35).

\section{Faire travailler le format}

Lorsqu'il finit par comprendre, au début des années quarante, que « The Church House Project » ne serait jamais réalisé faute de commanditaire, et sur les conseils de Dudley Tooth, son sage galeriste, Spencer se résolut à concevoir des œuvres séparées afin de pouvoir les vendre, renonçant au déploiement de la série. Spencer n'échappa pas aux rigueurs et aux nécessités de la réalité. Ceci fit évoluer les formats choisis à l'origine qui s'adaptèrent à la demande. En outre, lorsqu'un peintre conçoit une œuvre, il choisit une toile en fonction de son sujet. Il arrive alors que le format résiste et qu'il doive 
travailler avec lui, le faire travailler. Soit il faut le rallonger, soit il faut le raccourcir, ou encore le diviser, voire simplement l'adapter.

\section{Une nécessité interne : rallonger le format}

19 Ce fut le cas du spectaculaire tableau grouillant de personnages, Love on the Moor (79,1×310,2 cm, 1937-1955) conservé au Fitzwilliam Museum de Cambridge (Bell 1980, $219)^{20}$. Comme dans A Village in Heaven $(45,7 \times 182,9 \mathrm{~cm})$, l'action se déroule sur la lande de Cookham et prend pour sujet, dans Love on the Moor, l'apothéose d'Hilda et, dans A Village in Heaven ${ }^{21}$, une fête au village. La joie domine. Les deux œuvres sont de longs rectangles historiés et plus particulièrement encore Love on the Moor avec ses 3,10 mètres de long. En 1937, découragé devant le désintérêt de la critique, Spencer avait abandonné cette œuvre restée au stade du dessin. En 1949, l'artiste reprit Love on the Moor, resté à Cookham, commençant d'abord par faire un petit tableau de $76,2 \times 63,5$ $\mathrm{cm}^{22}$. Puis, il reprit le travail commencé en 1937, le termina et décida de le rallonger sur la gauche en lui adjoignant la partie qu'il venait d'exécuter. On peut voir la ligne de raccord verticale qui traverse le bout du mur de briques en haut de la composition. Le petit tableau, faisant désormais partie de l'œuvre, avait suffi à relancer le projet et à modifier le programme narratif.

De même, travaillant à The Resurrection: Port Glasgow (Bell 1980, 203) ${ }^{23}$, Spencer ne rajouta pas moins de 2,70 mètres à l'œuvre qui totalise $2,15 \mathrm{~m} \times 6,65 \mathrm{~cm}$ (Tate Gallery). Il $\mathrm{y}$ avait là comme une nécessité interne ${ }^{24}$. À l'origine, le tableau ne devait faire que les deux tiers de la taille actuelle mais, en 1949, Spencer y inclut une scène contenue dans l'un de ses carnets de dessins, celle de la tombe tabulaire qu'il peignit à gauche du tableau, alors presque terminé. Cependant, le centre de l'œuvre se trouvait déporté vers la gauche et la composition déséquilibrée. Spencer rajouta donc plus de deux mètres de toile, replaçant la tombe au centre de la composition. Les deux mondes représentés et réunis dans le vieux couple formé par Hilda et Spencer, agenouillés au milieu, au premier plan (Bell 1980, 202) s'en trouvaient équitablement répartis. Là encore, on distingue une division tripartite: au centre des groupes de villageois déchiffrant les inscriptions sur la tombe et de chaque côté deux mondes différents unis par les deux figures centrales. Comme on le verra plus loin, le triptyque est aussi un format affectionné par le peintre dans la tradition de ses maîtres anciens italiens.

\section{Raccourcir et/ou diviser le format}

21 Si Spencer rallongea le format de cette œuvre emblématique, à l'origine, il avait dû diviser le projet d'ensemble et, pour des raisons commerciales, renoncer à son projet initial et découper les longues frises en séries afin de pouvoir les vendre. Lors de son séjour à Port Glasgow, travaillant à la commande passée par le MOI (Ministère de l'Information) pendant la Seconde Guerre mondiale, Spencer fut inspiré par la vision de la forme de la colline du cimetière et conçut alors le projet d'une vaste frise de quinze mètres sur son thème favori, celui de la Résurrection. Le Christ devait siéger au centre, rendant son Jugement depuis la colline de Sion, se détachant sur le ciel et une armée d'anges. Émergeant en dessous, les morts seraient sortis de terre et se seraient avancés pour être jugés. On voit, là encore, l'importance des textes sacrés dans le processus créatif de Spencer et son inspiration. Finalement, craignant l'échec, Spencer adopta le compromis déjà utilisé pour « The Church House » : il divisa la composition en une série 
de tableaux autonomes et proposa alors, sous la forme d'une série, une variation du thème sur un mode plus prosaïque et familier que les versions canoniques. Spencer put conserver quand même The Resurrection: Port Glasgow (1945-1950), elle-même de $2,15 \times 6,65 \mathrm{~cm}$, The Hill of Zion $(94 \times 190,5 \mathrm{~cm}, 1946)$ et Angels of the Apocalypse $(70 \times 88,9 \mathrm{~cm}$, 1949) qui correspondent à peu près aux trois thèmes initiaux de la frise de départ mais désormais séparés en trois œuvres distinctes. Les autres œuvres de la série qui vinrent s'ajouter variaient la thématique déclinée selon les humeurs des participants : réjouissances, nettoyage, éveil, réunions de familles, résurrection des parents, etc. Ils sont de dimensions beaucoup plus modestes comparées aux 6,65 mètres du magistral The Resurrection: Port Glasgow, avec ses 2,70 mètres de rallonge. On voit là comment, au cours de l'élaboration d'une œuvre, le format peut varier considérablement et le tableau subir des coupures et des rajouts.

On pourrait citer d'autres exemples comme The Promenade of Women, qui aurait dû appartenir à la même frise que A Village in Heaven, conçue à l'origine pour «The Church House ». Ou encore le projet de frise répondant à une commande passée en 1929 par The Empire Marketing Board sur le thème de «Industry and Peace " pour des affiches qui auraient ensuite été distribuées dans tout le pays. Le peintre proposa de réaliser deux longues frises de 3,69 mètres chacune mais elles furent refusées car trop longues, si bien que la première fut divisée en cinq tableaux. Trois d'entre eux furent montés en triptyque pour l'exposition de la Tate Gallery dans les années trente, les deux autres étant présentés sur les côtés de la salle. Il s'agit, pour le triptyque central, de The Hat Stand, The Anthracite Stove et Cutting the Cloth. Les deux panneaux adjacents étaient The Life Class à gauche et The Garage à droite. Puis les œuvres furent dispersées alors que Spencer les avait conçues comme une unité25.

\section{Adapter le format : transferts}

Enfin, on notera le travail du peintre qui sut aussi adapter le format d'une manière originale. Il s'agissait non de réduire une frise mais de réutiliser, en l'agrandissant, une œuvre antérieure en la transférant d'un support à un autre. Ainsi, Spencer se servit de certaines de ses illustrations réalisées en 1926 pour une commande de l'Almanac de Chatto and Windus pour l'année 1927. Il puisa à ce fond tout prêt, plus tard, en adaptant certaines de ces vignettes, par exemple, pour le tableau de The Dustbin Cookham (1955) de dimensions modestes $(76,2 \times 50,6 \mathrm{~cm})$ qui, à l'origine, illustrait le mois de septembre 1927, ou le long rectangle de In Church $(61 \times 2,16 \mathrm{~cm}, 1958)$ qui illustrait le mois de juin 1927. Dans les Scènes domestiques, il adapta le format des vignettes pour composer Dusting the Shelves (1936), là aussi de dimensions modestes $(76 \times 51 \mathrm{~cm})$, et The Nursery or Christmas Stockings $(76,5 \times 91,8 \mathrm{~cm}, 1946)$. Spencer utilisa alors la technique traditionnelle de la mise au carreau qu'il pratiqua toute sa vie. La technique de carroyage permet la reproduction d'une scène ou d'une œuvre avec changement d'échelle et de proportions si nécessaire (Carline 12). Une célèbre gravure sur bois de Dürer, datant de 1525 et conservée au Rijksmuseum d'Amsterdam, montre le procédé qui consiste à réduire une scène ou une œuvre précédente, voire un modèle nu, en l'occurrence, face à une grille composée de carrés que l'artiste reporte sur la feuille destinée à l'œuvre à réaliser. 


\section{Les triptyques et les polyptiques : l'espace des figures de son imaginaire} forme canonique du triptyque, semblent avoir été parmi ses favorites. Elles devaient répondre à une manière personnelle de penser et d'organiser l'espace et l'histoire, une sorte de chronotope intime. En outre, le triptyque lui procurait un espace suffisant lui permettant d'articuler et de déployer les figures de son imaginaire ainsi que ses thèmes favoris de manière à les conserver et les faire jouer ensemble. Il avait appris à ses dépens que le recours au format de la série risquait d'entrainer la dispersion des tableaux et en compromettre la cohérence d'ensemble. La série des Domestic Scenes et celle des Beatitudes of Love se trouvent désormais disséminées dans diverses collections privées non visibles du grand public, à l'exception notable de certaines d'entre elles, comme de The Nursery or Christmas Stockings, qui se trouve au MoMA de New York, ou encore The Beatitudes of Love: Contemplation conservée à la Stanley Spencer Gallery de Cookham.

Cependant, Spencer choisit de combiner les deux types de formats, réalisant des séries de polyptyques, lorsqu'en 1939 le Ministère de l'Information lui passa commandes de cinq œuvres destinées soutenir l'effort de guerre et valoriser le travail des ouvriers dans les chantiers navals de Glasgow, sur la Clyde. Spencer se rendit à Port Glasgow et à Lithgow et fut enthousiasmé par ce qu'il y vit ainsi que par l'accueil chaleureux qu'il y reçut. Les cinq œuvres prirent la forme d'une série de polyptyques aux dimensions immenses dont le monumental Shipbuilding on the Clyde (1940-1946) conservé à l'Imperial War Museum de Londres $^{26}$. Les dimensions des toiles et les couleurs, les attitudes des ouvriers, les détails de leurs vêtements, la variété de leurs outils, sont assemblés en des scènes à l'éclairage caravagesque. Spencer utilise les forts contrastes entre l'ombre et la lumière des ateliers et des chantiers navals pour ses œuvres où vacillent et flamboient toutes sortes de scènes étranges. La célébration du travail prend la forme de scènes quasi-religieuses.

Trois longs panneaux d'environ cinq mètres chacun, comportant au milieu une structure élevée d'environ un mètre, forment des triptyques dédiés aux différents corps de métier: Shipbuilding on the Clyde: Burners, Shipbuilding on the Clyde: Welders, Shipbuilding on the Clyde: Riggers ${ }^{27}$. Shipbuilding on the Clyde: Plumbers fut faussement référencé comme prédelle du panneau central en 1944 alors qu'il ne comportait que deux longs rectangles sans centre élevé. La seule prédelle réalisée pour ces triptyques fut la très longue bande de The Template $(50,7 \times 5,79 \mathrm{~cm})$ destinée à être accrochée sous Burners. Les autres œuvres sont aussi d'un format oblong, longues d'environ cinq mètres: Shipbuilding on the Clyde: Riveters $(76,2 \times 579,2 \mathrm{~cm})$; Shipbuilding on the Clyde: Bending the Keel Plate $(76 \times 5,75 \mathrm{~cm})$. Spencer ajouta d'autres œuvres à ces commandes comme le magnifique clair-obscur de Furnaces, qui représente la sortie du métal en fusion des hauts fourneaux, extrait par les ouvriers tendus dans l'effort tout autour. Verticale, de dimensions plus sobres $(158 \times 114 \mathrm{~cm})$, cette œuvre conçue pour occuper le centre du dispositif est vue en plongée. Cette perspective était un parti pris esthétique affectionné par Spencer comme dans une autre belle œuvre de guerre : Travoys Arriving with Wounded at a Dressing Station at Smol, Macedonia, September 1916 $6^{28}$. Furnaces ${ }^{29}$ rougeoie de toutes ses couleurs projetées sur les visages et les corps des hommes au travail disposés en étoile autour du fleuve de lumière. 

hommes unis dans l'effort de guerre, une œuvre dont on peut aussi contempler les nombreux dessins effectués in situ par le peintre. Des photographies le montrent entouré d'ouvriers regardant ses croquis. À noter qu'on est loin des œuvres froides de propagande de la même époque commandées par des régimes autoritaires. L'humanité émanant du profond lien que Spencer avait noué avec les habitants de Port Glasgow transparait dans le respect et l'admiration du travail effectué sous ses yeux, lorsqu'il loue l'habileté des mains des ouvriers. Il s'est même représenté dans la partie gauche du triptyque intitulé Welders, le visage abrité derrière un masque de soudeur avec des pinceaux sortant de sa poche ${ }^{30}$. À noter aussi, comme le remarque Bell, que cette série fut la seule à correspondre au projet original dans sa forme (Bell 1980, 181). cinq prédelles prévues dans le projet à être terminée, c'est que, dès 1943, Spencer fut subjugué par la vision du cimetière de port Glasgow qui donna la série de The Resurrection: Port Glasgow dont le format de plus de cinq mètres pour le panneau éponyme est comparable au format des polyptyques de Shipbuilding on the Clyde. Spencer devait mener de front les deux séries et ne termina la commande officielle qu'en 1946 sous la pression. Une fois de plus, il était inspiré par le thème de la Résurrection des corps, un thème d'espoir dont il ne cessa de donner des versions (Louvel 2014). La persistance du thème, qui revient comme une hantise, correspond probablement au traumatisme de la guerre et aux visions des nombreux jeunes corps gisant sur les champs de bataille. Les équipes qui venaient les relever, dont Spencer faisait partie puisqu'il était brancardier, devaient faire face à l'horreur. Subsistent des photographies des corps alignés sous les croix blanches (Gough 2013, 168), comme celles qui structurent The Resurrection of Soldiers de Burghclere, reposant sur les corps avant qu'ils ne soient enterrés dans les cimetières militaires. Tous les pays européens, leurs alliés et leurs empires coloniaux, furent plongés dans le deuil et vécurent l'épreuve du fantasme du retour de ces jeunes soldats. Ceci correspondit pendant longtemps à une profonde aspiration, proche d'un rêve collectif de résurrection. C'est ce que Spencer transpose, en en peignant une version festive dans The Resurrection with the Raising of Jairus's Daughter (1947), l'un des tableaux de la série de The Resurrection: Port Glasgow qui s'inspire d'un épisode de l'Évangile, le Christ rappelant une enfant d'entre les morts (Marc 5: 22-43). Dans ce triptyque, il insère l'épisode dans le panneau central et dispose, à gauche, la résurrection des villageois qui sortent de sous les trottoirs du village et, à droite, leur accueil par les familles et la fête des retrouvailles. Voici la revanche de la vie sur la mort en trois panneaux. La Résurrection de Port Glasgow fut la dernière des résurrections peinte par Spencer, qui mourut en 1959.

Finalement, on pourrait presque dire que le thème de la résurrection a constitué comme une majeure série d'ensemble, trouvant son format ultime dans ses multiples déclinaisons y compris dans le tableau inaugural de Cookham (1924-26) puisqu'elle comporte trois parties distinctes qui constituent aussi un triptyque. Pala d'autel, avec ses trois niveaux, et triptyque avec ses trois scènes, l'œuvre réalisée après la guerre est l'une des plus célèbres de Spencer (et de la Tate Britain).

30 La récurrence de formats tels que les longs panneaux permettant de développer des scènes multiples dans un espace unifié et l'agencement des œuvres en triptyque témoignent d'un esprit original en pleine première moitié de ce $\mathrm{XX}^{\mathrm{e}}$ siècle, qui a vu tant d'innovations et aussi tant de catastrophes. Spencer, restant fidèle à son goût des 
maîtres anciens, a réussi à innover à sa manière et à mettre sous les yeux de ses contemporains parfois choqués, l'originalité de son imaginaire sans concession au bon goût. Ce fut le cas d'Edward Marsh, pourtant l'un de ses fidèles acheteurs, dont Maurice Collis rapporte que, perplexe devant les déformations des Beatitudes of Love, il demanda au peintre : "Oh Stanley, are people really like that?' I said: 'What's the matter with them? They are all right aren't they?' 'Terrible, terrible, Stanley!' 'Poor Eddie'» (Collis 144, cité dans Bell 1980, 167). On voit alors combien non seulement les thèmes choisis, mais aussi les conditions matérielles de leur "mise en œuvre", une chapelle, un mur, des formats anciens, jouent un rôle prépondérant dans le processus créatif. Bien entendu, Spencer dut s'adapter au goût de ses connaisseurs potentiels, poussé aussi par Dudley Tooth, et réalisa de nombreux tableaux dans des formats plus classiques, comme les beaux paysages très appréciés qui annoncent déjà ceux de David Hockney, ou bien de surprenants nus tels ceux de Patricia Preece ou de lui-même posant avec elle. Ces œuvres dont la « manière » étonnante, toute de chair luminescente et sans concession, a probablement influencé Lucian Freud. Il n'est pas jusqu'à Francis Bacon qui, lui aussi, semble avoir hérité de Spencer la distorsion des figures et le format du triptyque. Ce peintre, qui a pu sembler anachronique, allait donc étonnement inspirer ses modernes descendants. Alors, lorsque son amie, Elizabeth Rothenstein, déclarait comme on l'a vu plus haut, qu'il était un artisan médiéval et un simple "village boy ", on voit que, si elle avait en partie raison, elle n'avait pas pressenti-mais elle écrivait cela en 1945- son apport à la peinture de son temps et à celle qui allait venir avec les jeunes peintres de la future École de Londres.

\section{BIBLIOGRAPHIE}

\section{Ouvrages cités}

ABBOTT, Claude Colleer et Anthony BERTRAM (dir.). Poet and Painter. Being the Correspondence between Gordon Bottomley and Paul Nash 1910-1946. Oxford : Oxford University Press, 1955.

BEDDINGTON, Jenny et Jamie Hacker HUGHES. « Finding Heaven in a Hell of War: A Psychological Perspective ». Heaven in a Hell of War (catalogue de l'exposition de Somerset House, Londres). Dir. Amanda Bradley and Howard Watson. Londres : National Trust, 2013. 63-69.

BELL, Keith. Stanley Spencer, RA (catalogue de l'exposition à la Royal Academy of Arts, Londres). Londres : Royal Academy of Arts London; Weidenfeld \& Nicolson, 1980.

BELL, Keith. Stanley Spencer: A Complete Catalogue of the Paintings. Londres : Phaidon Press, 1992. BOLTER Jay David et Richard GRUSIN. Remediation: Understanding New Media. Cambridge MA: The MIT Press, 1999.

BRADLEY, Amanda. «The Reluctant Maecenases: John Louis and Mary Berhend ». Heaven in a Hell of War (catalogue de l'exposition à Somerset House, Londres). Dir. Amanda Bradley et Howard Watson. Londres : National Trust, 2013. 19-29. 
BRADLEY, Amanda et Howard WATSON (dir.). Heaven in a Hell of War (catalogue de l'exposition à Somerset House, Londres). Londres : National Trust, 2013.

CARLINE, Richard. « Stanley Spencer: His Personality and mode of Life ». Stanley Spencer (catalogue de l'exposition à la Royal Academy of Arts, Londres). Londres : Royal Academy of Arts London; Weidenfeld \& Nicolson, 1980. 9-18.

CAUSEY, Andrew. «Stanley Spencer and the Art of his Time ». Stanley Spencer RA (catalogue de l'exposition à la Royal Academy of Arts, Londres), Londres : Royal Academy of Arts ; Weidenfeld \& Nicolson, 1980. 19-36.

CHASTEL, André. Histoire du retable italien, des origines à 1500. Paris : Liana Levi, 2005.

COLLIS, Maurice. Stanley Spencer. Londres : Harvill Press, 1962.

DURING, Élie. « Le Monde doit être maquettisé ». In Octavo. Des formats de l'art. Dir. David Zerbib. Dijon : ESAAA Éditions / Les Presses du Réel, 2015. 23-48.

FRY, Roger. « Mr Frank Dodson and Mr. Stanley Spencer. ». Nation and the Atheneum (12 March 1927) : n.p.

GOUGH, Paul. Stanley Spencer, Journey to Burghclere. Bristol : Samson and Company, 2006.

GOUGH, Paul. «'That vile place': The Beaufort War Hospital, Bristol, as a Source of Inspiration for Stanley Spencer ». Heaven in a Hell of War (catalogue de l'exposition à Somerset House, Londres). Dir. Amanda Bradley et Howard Watson. Londres : National Trust, 2013. 9-17.

LOUVEL, Liliane. Le Tiers pictural. Pour une critique intermédiale. Rennes : Presses Universitaires de Rennes, 2010. The Pictorial Third: An Essay into Intermedial Criticism. Trad. en anglais Angeliki Tseti. Londres : Routledge, 2018.

LOUVEL, Liliane. «Repellent Shapes and Bewildering 'illustrations': Stanley Spencer's eccentric Styles ». Interfaces 37 (2017b, « Appropriation et réappropriation des récits », dir. C. Cambray, C. Marcangeli, S. Prévost, M. Géracht, B. Smith) : 117-128.

LOUVEL, Liliane, « Stanley Spencer: A Very Private Memorial ». War Memories: Commemoration, Recollections and Writings on War. Dir. Stéphanie Bélanger et Renée Dickason. Montréal, Kingston, Londres, Chicago : McGill-Queen's University Press, 2017a. 176-293.

LOUVEL, Liliane. Stanley Spencer. Un Visionnaire du quotidien. Paris : PUP Sorbonne, 2021.

LOUVEL, Liliane. «The Resurrections of Stanley Spencer ». Interfaces 35 (2014, « Re-readings and Re-Viewings of Sacred/Archetypal Narratives in Literature and the Arts », dir. M. Geracht et B. Smith) : 81-104.

MARTIN, Simon. «'What Ho Giotto': Stanley Spencer's Holy Box and the Influence of the Italian Primitives". Heaven in a Hell of War (catalogue de l'exposition à Somerset House, Londres). Dir. Amanda Bradley et Howard Watson. Londres : National Trust, 2013. 53-63.

MERLEAU-PONTY, Maurice. « Le langage indirect ». La Prose du monde. Paris : Gallimard (coll. « Tel »), 1969. 66-160.

MONCOND’HUY, Dominique. «La galerie et sa ‘description' en France : le modèle Richelieu ». Suite, série, séquence. Dir. Dominique Moncond'huy et François Noudelmann. La licorne (1998) : 21-35. URL : https://licorne.edel.univ-poitiers.fr/index.php?id=191 (consulté le 21 mai 2021). MONCOND'HUY, Dominique et François NOUDELMANN (dir.). Suite, série, séquence. La licorne (1998) : 21-35. URL : https://licorne.edel.univ-poitiers.fr/index.php?id=191 (consulté le 21 mai 2021). 
NORRIS, Katy. « Masculinity and Fraternity in Stanley Spencer's Vision of War ». Heaven in a Hell of War (catalogue de l'exposition à Somerset House, Londres). Dir. Amanda Bradley et Howard and Watson. Londres : National Trust, 2013. 41-51.

ORTEL, Philippe. Discours, image, dispositif, Penser la représentation II. Paris : L’Harmattan (« champs visuels »), 2008.

ROTHENSTEIN, Elizabeth. Stanley Spencer. Oxford, Londres: Phaidon Press, 1945.

STOICHITA, Victor. L'Effet Sherlock Holmes. Variations du regard de Manet à Hitchcock. Paris : Hazan, 2015.

STOICHITA, Victor. L'Instauration du tableau. Métapeinture à l'aube des temps modernes. Genève : Droz, 1999.

TAYLOR, David. «The Sun to Spencer's Planet: Henry Lamb and Stanley Spencer ». Heaven in a Hell of War (catalogue de l'exposition à Somerset House, Londres). Dir. Amanda Bradley et Howard and Watson. Londres : National Trust, 2013. 31-39.

VAUGEOIS, Dominique. « Le Rythme du livre : le sériel et les lieux du lisible dans Henri Matisse, Roman ». Suite, série, séquence. Dominique Moncond'huy et François Noudelmann. La licorne (1998) : 301-311. URL : https://licorne.edel.univ-poitiers.fr/index.php?id=191 (consulté le 21 mai 2021).

ZERBIB, David. «Introduction : Voulez-vous enregistrer les modifications ? ». Octavo. Des formats de l'art. Dir. David Zerbib. Dijon : ESAAA Éditions/Les Presses du Réel, 2015a.13-19.

ZERBIB, David. «1. Échelles et représentations ». Octavo. Des formats de l'art. Dir. David Zerbib. Dijon : ESAAA Éditions/Les Presses du Réel, 2015b. 21.

\section{NOTES}

1. Pour un large aperçu des œuvres de Spencer on consultera :

https://www.wikiart.org/en/stanley-spencer

(consulté le 21 mai 2021).

2. Si Zerbib s'intéresse d'abord aux pratiques plastiques contemporaines, ses remarques sont valables pour un peintre tel que Spencer qui a bien fait subir aux textes sacrés, et aux images qui les avaient illustrés précédemment, des opérations de traduction, de transposition, de remédiation et autres transformations intermédiales.

3. https://stanleyspencer.org.uk est le site de la Galerie de peinture dédiée à Stanley Spencer dans son village de Cookham. Elle est abritée dans l'ancienne chapelle méthodiste où l'artiste et ses frères et sœurs assistaient à l'office du dimanche accompagnés par leur mère. Sa maison natale est à deux pas dans la même rue.

4. Il choisit d'y inscrire l'une des œuvres pour lesquelles il est célèbre, The Resurrection, Cookham (1924, Tate Gallery) qui se déroule dans le cimetière de Cookham qui n'a guère changé depuis.

5. Stanley Spencer devint Sir Stanley Spencer O.B.E. en 1959, l'année de sa mort.

6. Ce que l'on nomme pour suivre Bolter et Grusin (1999) une opération de remédiation.

7. Dans ce passage, Dominique Vaugeois se réfère à sa discussion précédente de la définition de «l'ontologie du tableau moderne» selon Maurice Merleau-Ponty (Merleau-Ponty 77). 
8. Il s'agit de l'œuvre conservée à la Tate Gallery, commencée juste avant de partir à la guerre et qu'il put terminer, avec quelle joie !, en rentrant. http://www.wikiart.org/ en/stanley-spencer/swan-upping-at-cookham_(consulté le 21 mai 2021).

9. Le lieutenant Sandham était revenu de la guerre sévèrement affecté du paludisme dont Spencer et l'un de ses frères souffrirent également. Dans certaines des scènes de Burghclere, on voit les moustiquaires protectrices et les malades en proie aux fièvres du paludisme.

10. Clémenceau appela sarcastiquement les soldats français postés sur ce front des Balkans jugé marginal, «les jardiniers de Salonique» car, pour lutter contre la dysenterie, le paludisme et le scorbut, ils durent assécher les marais et cultiver la terre. Voir http://www.souvenir-francais-92.org/article-les-jardiniers-desalonique-69683379.html (consulté le 21 mai 2021).

11. De novembre 2013 à janvier 2014, les tableaux des côtés peints sur toile ont été exposés à Somerset House, Londres, pour commémorer la Grande Guerre. L'exposition "A Heaven in a Hell of War ", reconstituait la chapelle et on y voyait les œuvres des côtés disposées de la même façon que dans le Memorial. Une projection vidéo rendait présentes les scènes qui n'avaient pas pu être déposées : celles du haut et la grande Résurrection des soldats. Il s'agissait de montrer l'œuvre de Spencer pendant la restauration du lieu puisque le climat anglais humide n'est pas vraiment propice à une conservation de fresques, dussent-elles être sur toile. À noter que si ce type de décoration est assez rare en Angleterre, il y en eut d'autres exemples plus tard. Pendant la Seconde Guerre mondiale, Vanessa Bell et Duncan Grant décorèrent ainsi la chapelle de Berwick, Sussex, non loin de leur maison de Charleston et de Monk's House où vivaient Virginia and Leonard Woolf. La décoration s'inspira de thèmes religieux traditionnels comme celui de l'Annonciation, de la Nativité ou de la Crucifixion. Les artistes empruntèrent néanmoins leurs visages à leurs proches comme celui d'Angelica Bell, fille de Vanessa et de Clive Bell, et de l'un des amis proches de Duncan Grant, entre autres, ce qui ne manqua pas de choquer les paroissiens. À noter que dans la chapelle de Burghclere les thèmes religieux traditionnels ne sont pas traités. Il s'agit de scènes profanes à l'exception de la Résurrection des soldats. On se rendra sur le beau site de la Sandham Memorial Chapel qui est désormais la propriété du National Trust pour voir l'architecture du lieu et les vues intérieures. Voir https://www.nationaltrust.org.uk/ sandham-memorial-chapel (consulté le 21 mai 2021). Voir aussi le catalogue de Bradley et Watson (2013). Voir le catalogue de l'exposition de Somerset House, Londres : Heaven in a Hell of War, dir. Amanda Bradley and Howard Watson. Londres : National Trust, 2013, qui comporte de nombreux articles cités dans la bibliographie.

12. Chastel insiste aussi d'ailleurs sur le lien entre l'architecture, le cadre, l'architectonique et la composition: "La pala intègre ce qui préexistait à la composition » note-t-il. Et plus loin «le cadre de la pala d'autel est de plus en plus souvent assorti à celui de son habitacle » $(70,112)$.

13. Elizabeth et John Rothenstein, ce dernier alors directeur de la Tate Gallery, hébergèrent Spencer un temps à la suite de ses déboires consécutifs à son second mariage.

14. Nous avons des dessins de Spencer montrant Hilda, sa première femme, allongée sur le sol dans l'un de ses cartons et regardant les dessins projetés sur ce qui serait les murs. 
15. Double Nude Portrait (1937, Tate Gallery) : https://www.pinterest.com/pin/ 459859811924812083/ ; Self Portrait with Patricia Preece (1936, Fitzwilliam Museum Cambridge :

http://www.fitzmuseum.cam.ac.uk/pharos/collection_pages/20th_pages/PD.966-1963/ PIC_SE-PD.966-1963.html_(pages consultées le 21 mai 2021).

16. «Mr Spencer is a literary painter, he works by imagery. Our quarrel with such should not be that they are not plastic-they are practising an alternative art-but that, for the most part, their imagery is so dull and inexpressive. That complaint cannot be urged against The Resurrection...It is no perfunctory or sentimental piece of storytelling, but a personal conception carried through with unfailing verve and conviction. And such imagery is not so common but that it should be welcomed even by those who, like myself, find its total effect to be a distinctly unpleasant and disagreeable stimulation » (Fry, Nation and Atheneum, cité par Bell, 1992, 67).

17. Voir https://stanley-spencer.tumblr.com/post/189443774788/the-marriage-atcana-bride-and-bridegroom (consulté le 21 mai 2021) : sur ce site plusieurs œuvres de Spencer sont reproduites, outre une scène du mariage et une autre des Béatitudes.

18. Sur les Domestic Scenes voir https://www.bing.com/images/search? $\mathrm{q}=$ stanley+spencer+domestic+sc\%c3\%a8nes\&qpvt=Stanley+Spencer+Domestic+Scenes\&form=IGRE\&first=1\&tsc=ImageBasic (consulté le 21 mai 2021).

19. https://www.wikiart.org/en/stanley-spencer/beatitudes-of-love-8-worship-1938 (consulté le 21 mai 2021).

20. https://www.artuk.org/discover/artworks/love-on-the-moor-4600 (consulté le 21 mai 2021).

21. https://www.artuk.org/discover/artworks/a-village-in-heaven-206089 (consulté le 21 mai 2021).

22. L'un des facteurs déclenchants de cette reprise fut l'accusation portée par Sir Alfred Munnings, alors président de la Royal Academy of Arts, d'être un peintre pornographique (Bell 1980, 219). L'œuvre terminée fut rapidement achetée par Wilfrid Ewill, grand collectionneur.

23. https://www.tate.org.uk/art/artworks/spencer-the-resurrection-port-glasgown05961 (consulté le 21 mai 2021).

24. C'est la seule de la série des Resurrection: Port Glasgow à avoir la dimension prévue initialement par l'artiste, soit 2,15×6,65 cm, avec the Hill of Zion $(94 \times 190,5 \mathrm{~cm})$.

25. L'exposition de 1980, pour la première fois, présentait les œuvres réunies (Bell 1980, 114-18).

26. On en trouve des reproductions ainsi que des autoportraits dans l'article de wiki art: https://en.wikipedia.org/wiki/Stanley_Spencer. Une vidéo sur le site https:// www.dailymotion.com/video/x6eqjto montre l'artiste lui-même au travail. Autres vidéos : https://www.youtube.com/watch?v=U9zc0fdNxi0; Welders (1941) https:// www.iwm.org.uk/collections/item/object/25150; Furnaces : (1946) https:// artsandculture.google.com/asset/shipbuilding-on-the-clyde-the-furnaces/ aAEOXiTjl_HYOA (pages consultées le 21 mai 2021).

27. Shipbuilding on the Clyde: https://www.iwm.org.uk/collections/item/object/25150 (consulté le 21 mai 2021). Les images de la série sont accessibles comme celle de Welders (numéro 25150), par exemple, sur le site de l'IWM. D'autres sites et vidéos détaillent les œuvres. 
28. Voir https://www.iwm.org.uk/collections/item/object/25132 (consulté le 21 mai 2021).

29. Voir https://artsandculture.google.com/asset/shipbuilding-on-the-clyde-thefurnaces/ (consulté le 21 mai 2021).

30. Cette pratique de l'insertion d'un autoportrait du peintre dans une œuvre à plusieurs personnages est bien une technique remontant à la Renaissance italienne et pratiquée par exemple par Benozzo Gozzoli, Raphaël, Michel-Ange, etc.

\section{RÉSUMÉS}

La question du format s'est posée pour Stanley Spencer de manière cruciale concernant le processus créatif de ses œuvres avec figures. Il témoigne d'une affinité comme anachronique pour les séries, la mise en triptyques de ses œuvres, un goût pour les longs formats étroits, lui permettant de dérouler les séquences d'un récit. Inspiré par les Primitifs italiens, il décora «à fresque " la chapelle de Burghclere avec ses souvenirs de guerre et poursuivit ce rêve d'une totalité alliant architecture et peinture avec "The Church House Project» qui ne rencontra jamais de commanditaire. Mais les séries prévues sont là pour en témoigner. La commande officielle, pendant la Seconde Guerre mondiale, d'aller sur les chantiers navals de Glasgow, permit à Spencer de peindre de nouvelles séries extraordinaires. Soumis aux conditions de production, le peintre dut aussi parfois jouer avec/contre le format, l'allongeant, le réduisant, voire le transposant. Ces altérations témoignent de la nécessité de travailler avec le réel, dût-elle entraîner des sacrifices.

For Stanley Spencer formatting was always linked to his own « inner vision » and inspiration. It reveals a kind of anachronistic taste for series, triptychs and long narrow formats allowing him to paint narrative sequences with figures. Deeply inspired by the Italian Primitive painters, Spencer decorated the famous Burghclere Chapel with frescoes evoking his war memories and hoped to renew this major feat with "The Church House Project", but he never found a commissioner. Fortunately its several series display his creativity. The War commission sending him to Glasgow to paint Shipbuilding on the Clyde gave him once more the opportunity to produce several magnificent series. While having to cope with production constraints the painter often had to work with/against formats: adding to them, cutting them up, even adapting them to new projects. Thus these alterations testify to the necessity to working with reality, were it to entail painful sacrifices.

\section{INDEX}

Mots-clés : Spencer (Stanley), séries, triptyques, fresques, chapelle de Burghclere, « The Church House Project ", Shipbuilding on the Clyde, éthique, esthétique

Keywords : Spencer (Stanley), series, triptychs, frescoes, Burghclere Chapel, "The Church House Project", Shipbuilding on the Clyde, ethics, aesthetics 


\section{AUTEUR}

\section{LILIANE LOUVEL}

Université de Poitiers

Liliane Louvel est professeur émérite de littérature britannique à l'Université de Poitiers. Son champ de recherche est la littérature britannique contemporaine et les rapports texte/image. Elle a publié cinq ouvrages sur le sujet : L'œil du texte (Toulouse, PUM, 1998), The Picture of Dorian Gray. Le double miroir de l'art (Ellipses, 2000), Texte/image, images à lire et textes à voir (Rennes, PUR, 2002). Le tiers pictural. Pour une critique intermédiale (PUR, 2010) et Poetics of the Iconotext (Trad. Laurence Petit, ed. Karen Jacobs, Ashgate, 2011). Le tiers pictural, traduit par Angeliki Tseti avec une Préface de Julie Leblanc, a été publié sous le titre : The Pictorial Third (Routledge, 2018). Elle a publié de nombreux articles et dirigé plusieurs recueils d'essais sur le même sujet : Word/image (EJES) ; Like Painting (La licorne) ; les Actes du colloque de Cerisy Texte/image nouveaux problèmes avec Henri Scepi (PUR) ; Intermedial Arts, Disrupting, Remembering and Transforming Media avec Leena Eilitta et Sabine Kim (Cambridge Scholars Press, 2012) ; Musing in the Museum avec Laurence Petit et Karen Brown (Word \& Image, mai 2015). Elle est présidente d'honneur de la SAES et honorary president of ESSE. Elle est également présidente de IAWIS/AIERTI (International Society for Word \& Image Studies/Association Internationale pour les Rapports entre Texte et Image). 